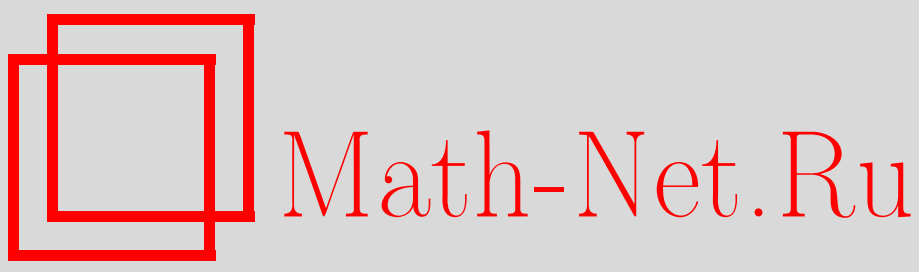

Б. С. Калитин, О проблеме Айзермана для скалярных дифференциальных уравнений, Изв. вузов. Матем., 2019, номер 9, 37-49

DOI: https://doi.org/10.26907/0021-3446-2019-9-37-49

Использование Общероссийского математического портала Math-Net.Ru подразумевает, что вы прочитали и согласны с пользовательским соглашением

http://www . mathnet.ru/rus/agreement

Параметры загрузки:

IP: 54.224 .187 .69

26 апреля 2023 г., $11: 37: 55$

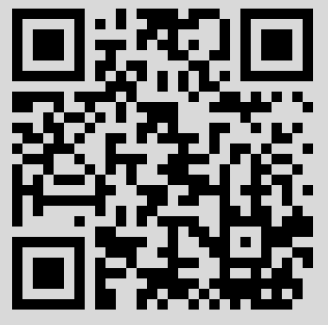


Известия вузов. Математика

2019, № 9, c. $37-49$ https://kpfu.ru/science/nauchnye-izdaniya/ivrm

e-mail: izvuz.matem@kpfu.ru

\title{
Б.С. КАЛИТИН
}

\section{О ПРОБЛЕМЕ АЙЗЕРМАНА ДЛЯ СКАЛЯРНЫХ ДИФФЕРЕНЦИАЛЬНЫХ УРАВНЕНИЙ}

\begin{abstract}
Аннотация. Рассматривается задача устойчивости равновесия скалярного дифференциального уравнения $n$-го порядка. Получено положительное решение проблемы Айзермана для уравнений специального вида. Доказано, что параметр действительной части корня характеристического уравнения можно заменить произвольной непрерывной функцией, зависящей от всех фазовых переменных с сохранением свойства глобальной асимптотической устойчивости.
\end{abstract}

Ключевые слова: скалярное дифференциальное уравнение, равновесие, устойчивость, функция Ляпунова.

УДК: 517.925

DOI: 10.26907/0021-3446-2019-9-37-49

\section{1. ВВЕДЕНИЕ}

Проблема М.А. Айзермана, связанная с задачами глобальной асимптотической устойчивости динамических систем, сформулирована в работе [1] для систем $n$ дифференциальных уравнений и привлекла внимание многочисленных исследователей к ее решению и обсуждению. Это работы Н.П. Еругина [2], [3], И.Г. Малкина [4], Н.Н. Красовского [5], В.А. Плисса [6], Г.А. Леонова [7] и др.

Для случая $n=2$ проблема исследована Н.П. Еругиным, указавшим многие случаи, когда имеется положительное решение.

Примеры Н.Н. Красовского [5] и В.А. Плисса [6] показывают, что высказанная проблема может не иметь положительного решения для систем обыкновенных дифференциальных уравнений.

В исследованиях Г.А.Леонова приведены классы систем, для которых проблема Айзермана не имеет положительного решения, и наряду с этим предложен ряд подходов к ее решению.

Определенный анализ проблемы дан в работах [8], [9].

Напомним постановку проблемы Айзермана для скалярных дифференциальных уравнений.

Пусть $n \in \mathbb{N}$ - натуральное число и $x(t)(x: \mathbb{R} \rightarrow \mathbb{R})$ - непрерывно дифференцируемая функция. Будем использовать следующие понятия производных высших порядков:

$$
\dot{x}=\frac{d x}{d t}, \quad \ddot{x}=\frac{d^{2} x}{d t^{2}}, \ldots, x^{(k)}=\frac{d x^{(k-1)}}{d t}, \quad k=\overline{1, n}\left(x^{(0)}=x\right) .
$$

Поступила в редакцию 24.07.2018, после доработки 19.12.2018. Принята к публикации 19.12.2018 
Рассмотрим линейное скалярное дифференциальное уравнение

$$
x^{(n)}+\sum_{i=1}^{k-1} a_{i} x^{(n-i)}+a_{k} x^{(n-k)}+\sum_{i=k+1}^{k-1} a_{i} x^{(n-i)}+a_{n} x=0, \quad x \in \mathbb{R},
$$

с постоянными коэффициентами $a_{i}, i \in \overline{1, n}$, где $1<k \leq n$. Наряду с этим рассмотрим нелинейное уравнение

$$
x^{(n)}+\sum_{i=1}^{k-1} a_{i} x^{(n-i)}+f_{k}\left(x^{(n-k)}\right)+\sum_{i=k+1}^{k-1} a_{i} x^{(n-i)}+a_{n} x=0, \quad x \in \mathbb{R},
$$

где $f_{k}: \mathbb{R} \rightarrow \mathbb{R}$ - непрерывная функция, обеспечивающая единственность решений во всем фазовом пространстве. Предположим, что согласно условиям Рауса-Гурвица [9], решение $x=\dot{x}=\ddot{x}=\cdots=x^{(n-1)}=0$ уравнения (1.1) асимптотически устойчиво для всех $a_{k}$, удовлетворяющих условию

$$
\alpha<a_{k}<\beta .
$$

Поставим следующий вопрос: будет ли решение $x=\dot{x}=\ddot{x}=\cdots=x^{(n-1)}=0$ уравнения (1.2) глобально асимптотически устойчивым, если выполнено обобщенное условие РаусаГурвица [9]

$$
\alpha<\frac{f_{k}\left(x^{(n-k)}\right)}{x^{(n-k)}}<\beta, \quad x^{(n-k)} \neq 0 ?
$$

Покажем на примере скалярного дифференциального уравнения, что здесь так же проблема может не иметь положительного решения.

Пример 1.1. Рассмотрим задачу об устойчивости решения нелинейного скалярного дифференциального уравнения второго порядка

$$
\ddot{x}+f(x) \dot{x}+f(x) x=0,
$$

где

$$
f(x)= \begin{cases}e^{-x}\left(1+x e^{x}\right)^{-1}, & \text { если } x \geq 1 \\ e^{-1}(1+e)^{-1}, & \text { если } x<1\end{cases}
$$

Соответствующее линейное уравнение принимает вид

$$
\ddot{x}+a \dot{x}+a x=0 .
$$

Условия Рауса-Гурвица для такого линейного уравнения сводятся к неравенству $a>0$. Поэтому для нелинейного уравнения (1.3) соответствующее условие выполняется.

Из (1.3) получаем нелинейную систему уравнений

$$
\dot{x}=y, \quad \dot{y}=-x f(x)-f(x) y .
$$

Можно проверить, что $y=e^{-x}, x \geq 1$, есть уравнение части траектории для (1.4). Вдоль этой траектории имеем

$$
\dot{x}=e^{-x} .
$$

Следовательно, видим, что компонента $x(t)$ монотонно возрастает. Интегрируем это уравнение с начальными данными $x(0)=1$ и получим $x(t)=\ln (t+e), t \geq 0$. Поэтому вдоль траектории имеем $x(t)>1 \forall t \geq 0$ и, более того, $x(t) \rightarrow+\infty$ при $t \rightarrow \infty$. Следовательно, не смотря на то, что обобщенные условия Рауса-Гурвица выполняются для системы (1.4), дифференциальное уравнение (1.2) не является глобально асимптотически устойчивым. Таким образом, проблема Айзермана для скалярного дифференциального уравнения (1.3) не имеет положительного решения. 
В настоящей работе доказывается, что для скалярных дифференциальных уравнений определенного вида параметр вещественной части корня характеристического уравнения может быть заменен произвольной непрерывной функцией, зависящей от всех фазовых переменных с сохранением свойства глобальной асимптотической устойчивости. Для решения поставленной задачи используется метод знакопостоянных функций Ляпунова. Приведем для полноты изложения материала основные теоремы такого подхода.

\section{2. МЕТОД ЗНАКОПОСТОЯННЫХ ФУНКЦИЙ}

Пусть $\mathbb{R}^{n}$ - действительное $n$-мерное евклидово пространство со скалярным произведением $\langle\cdot, \cdot\rangle$ и нормой $\|\cdot\|=\sqrt{\langle\cdot, \cdot\rangle}$. Положим $B_{\varepsilon}=\left\{x \in \mathbb{R}^{n}:\|x\|<\varepsilon\right\}$ для $\varepsilon>0$.

Рассмотрим систему дифференциальных уравнений

$$
\dot{x}=f(x), \quad x \in \mathbb{R}^{n}, \quad f(0)=0,
$$

где $G$ - окрестность начала $0 \in \mathbb{R}^{n}$ и $f: G \rightarrow \mathbb{R}^{n}$ - непрерывная функция, удовлетворяющая условиям единственности решений в $G$. Решение $(2.1)$, проходящее через точку $x_{0} \in G$ в момент $t=0$, обозначим через $x\left(x_{0}, t\right)$, т. е. $x\left(x_{0}, 0\right)=x_{0}$. Система (2.1) обладает тривиальным решением $x=0$.

Будем использовать следующие определения [10], [11]. Множество $Y \subset \mathbb{R}^{n}$ называется положительно инвариантным, если $x\left(x_{0}, t\right) \in Y$ для каждого $x_{0} \in Y$ при всех $t>0$.

Пусть $Y$ - замкнутое связное положительно инвариантное множество в $\mathbb{R}^{n}$, содержащее начало координат. Решение $x=0$ системы (2.1) является

- $Y$-устойчивым, если

$$
(\forall \varepsilon>0)(\exists \delta=\delta(\varepsilon)>0)\left(\forall x_{0} \in B_{\delta} \cap Y\right) \Rightarrow\left\|x\left(x_{0}, t\right)\right\|<\varepsilon \forall t \geq 0 ;
$$

- $Y$-притягивающим, если

$$
(\exists \sigma>0)(\forall \alpha>0)(\exists T=T(\alpha)>0)\left(\forall x_{0} \in B_{\sigma} \cap Y\right) \Rightarrow\left\|x\left(x_{0}, t\right)\right\|<\alpha \forall t \geq T ;
$$

- $Y$-асимптотически устойчивым, если оно $Y$-устойчиво и $Y$-притягивающее;

- $Y$-глобально асимптотически устойчивым, если оно $Y$-асимптотически устойчиво, $G=$ $\mathbb{R}^{n}$, и $x\left(x_{0}, t\right) \rightarrow 0$ при $t \rightarrow+\infty$ для каждого $x_{0} \in Y$;

- неустойчивым, если

$$
(\exists \varepsilon>0)(\forall \delta>0)\left(\exists x_{0} \in B_{\delta}\right)\left(\exists t^{*} \geq 0\right) \Rightarrow\left\|x\left(x_{0}, t^{*}\right)\right\| \geq \varepsilon .
$$

Если $Y=\mathbb{R}^{n}$, тогда получаем обычные определения устойчивости, притяжения и асимптотической устойчивости [11].

Пусть $U$ - открытое подмножество $G, \bar{U}$ - замыкание $U$ и $\mathbb{R}^{+}-$множество неотрицательных чисел. Если $U \subset \mathbb{R}^{n}$ - открытое подмножество, то через $\mathbf{C}^{1}\left(U, \mathbb{R}^{+}\right)$обозначим множество всех непрерывно дифференцируемых функций $V: U \rightarrow \mathbb{R}^{+}$. Для $V \in \mathbf{C}^{1}\left(U, \mathbb{R}^{+}\right)$ через

$$
\dot{V}\left(x_{0}\right)=\frac{d V\left(x_{0}\right)}{d x}=\left\langle\frac{\partial V\left(x_{0}\right)}{\partial x}, f\left(x_{0}\right)\right\rangle
$$

обозначим производную по времени функции $V$ в силу системы $(2.1)$ в точке $x_{0}$.

Представим теоремы прямого метода Ляпунова с использованием знакопостоянных функций, начало которому положено работой [12].

Теорема 2.1 ([10]; [13], с. 58). Предположим, что для системы (2.1) существует окрестность $U$ точки $x=0$ и функиия $V \in \mathbf{C}^{1}\left(U, \mathbb{R}^{+}\right)$такал, ито

1) $V(x) \geq 0 \forall x \in U, V(0)=0$;

2) $\dot{V}(x) \leq 0 \forall x \in U$; 
3) $x=0$ асимптотически устойчиво относительно $Y_{0}=\{x \in \bar{U}: V(x)=0\}$. Тогда решение $x=0$ системы (2.1) устойчиво.

Теорема 2.2 ([10]; [12]; [13], с. 239). Предположим, что для системы (2.1) существует окрестность $U$ точки $x=0$ и функиия $V \in \mathbf{C}^{1}\left(U, \mathbb{R}^{+}\right)$такал, ито

1) $V(x) \geq 0 \forall x \in U, V(0)=0$;

2) $\dot{V}(x) \leq 0 \forall x \in U$;

3) решение $x=0$ асимптотически устойчиво относительно $Y=\{x \in \bar{U}: \dot{V}(x)=0\}$. Тогда решение $x=0$ системы (2.1) асимптотически устойчиво.

Теорема 2.3 ([10]; [12]; [13], с. 207). Пусть $G=\mathbb{R}^{n}$. Предположим, что для системъ (2.1) существует функция $V \in \mathbf{C}^{1}\left(\mathbb{R}^{n}, \mathbb{R}^{+}\right)$такая, что

1) $V(x) \geq 0 \forall x \in \mathbb{R}^{n}, V(0)=0$;

2) $\dot{V}(x) \leq 0 \forall x \in \mathbb{R}^{n}$;

3) решение $x=0$ глобально асимптотически устойчиво относительно множества $Y_{\infty}=\left\{x \in \mathbb{R}^{n}: \dot{V}(x)=0\right\}$

4) все решения системы (2.1) ограничены при $t \geq 0$.

Тогда решение $x=0$ системы (2.1) глобально асимптотически устойчиво.

Замечание 2.1. Следует иметь в виду, что при выполнении условий теорем 2.1, 2.2 или 2.3 неустойчивость относительно нетривиального множества $Y_{0}$ влечет неустойчивость относительно всего фазового пространства.

Для дальнейших исследований нам понадобится

Лемма 2.1 ([14], с.282). Пусть задана система дифференциальных уравнений $\dot{x}=A x+$ $f(t)$, где $A-$ постоянная $(n \times n)$-матрица, и $f: \mathbb{R}^{+} \rightarrow \mathbb{R}^{n}-$ непрерывная функция. Предположим, что

1) все характеристические числа $\lambda_{j}(A)$ матрицы $A$ имеют отрицателъные действительные части $\operatorname{Re} \lambda_{j}(A)<0, j=\overline{1, n}$;

2) функция $f(t)$ ограничена для $t>0$.

Тогда каждое решение системы $\dot{x}=A x+f(t)$ ограничено для $t>0$.

Доказательство. Для произвольного решения $x(t), x(0)=0$, заданной системы дифференциальных уравнений при $t>0$ справедлива формула ([14], с. 76)

$$
x(t)=e^{A t} x_{0}+\int_{0}^{t} e^{A(t-s)} f(s) d s, \quad t>0 .
$$

Отсюда можем записать неравенство для нормы решения

$$
\|x(t)\| \leq\left\|e^{A t}\right\|\left\|x_{0}\right\|+\int_{0}^{t}\left\|e^{A(t-s)}\right\|\|f(s)\| d s, t>0 .
$$

Заметим, что имеет место оценка $\left\|e^{A t}\right\| \leq N e^{-\alpha t}, t>0$, для нормы матрицы ([14], с. 282), где $N>0,0<\alpha<-\max R\left(\lambda_{j}(A)\right)$. Кроме того, по предположению ограниченности векторфункции $f(t)$ существует число $M>0$, для которого $\|f(t)\| \leq M$ при всех $t>0$. В результате получаем оценку для решения $x(t)$ в виде

$$
\|x(t)\| \leq N e^{-\alpha t}\left\|x_{0}\right\|+N M \int_{0}^{t} e^{A(t-\tau)} d s=N e^{-\alpha t}\left\|x_{0}\right\|+\frac{N M}{\alpha}\left(1-e^{-\alpha t}\right), \quad t>0 .
$$

Это и доказывает ограниченность решения $x(t)$. 


\section{3. ДИФФЕРЕНЦИАЛЬНОЕ УРАВНЕНИЕ $n$-ГО ПОРЯДКА}

Исследуем проблему устойчивости для тривиального решения $x=\dot{x}=\ddot{x}=\cdots=x^{(n-1)}=0$ нелинейного скалярного дифференциального уравнения (1.1) с функциональными коэффициентами, используя метод знакопостоянных функций Ляпунова.

Для случая постоянных коэффициентов в (1.1) характеристическое уравнение может быть записано в форме

$$
\lambda^{n}+\sum_{i=1}^{n-1} a_{i} \lambda^{n-i}+a_{n}=0
$$

1. Случай пары комплексно-сопряженных корней. Пусть $\lambda_{1}, \lambda_{2}-$ пара комплексносопряженных корней уравнения (3.1). Тогда это уравнение может быть записано следующим образом:

$$
\left(\lambda^{n-2}+\sum_{i=1}^{n-1} a_{i} \lambda^{n-i-2}+a_{n-2}\right)\left(\lambda^{2}+p \lambda+q\right)=0,
$$

где $p=-\lambda_{1}-\lambda_{2}, q=\lambda_{1} \lambda_{2}(p, q \in \mathbb{R})$.

Перемножая скобки в $(3.2)$, приходим к уравнению

$$
\begin{aligned}
\lambda^{n}+\left(\alpha_{1}+p\right) \lambda^{n-1}+\left(\alpha_{2}+p \alpha_{1}+q\right) \lambda^{n-2}+\sum_{j=3}^{n-2}\left(\alpha_{j}+\right. & \left.p \alpha_{j-1}+q \alpha_{j-2}\right) \lambda^{n-j}+ \\
& +\left(p \alpha_{n-2}+q \alpha_{n-3}\right) \lambda+q \alpha_{n-2}=0 .
\end{aligned}
$$

Сравнивая теперь представления (3.1) и (3.3), получаем равенства

$$
\begin{gathered}
a_{1}=\alpha_{1}+p, \quad a_{2}=\alpha_{2}+p \alpha_{1}+q, \\
a_{i}=\alpha_{i}+p \alpha_{i-1}+q \alpha_{i-2}, \quad i=\overline{3, n-2}, \\
a_{n-1}=p \alpha_{n-2}+q \alpha_{n-3}, \quad a_{n}=q \alpha_{n-2} .
\end{gathered}
$$

Заметим, что характеристическое уравнение любого линейного дифференциального уравнения может быть записано в виде (3.3).

Представление (3.2) показывает, в частности, что согласно условиям Рауса-Гурвица для асимптотической устойчивости решения $x=\dot{x}=\ddot{x}=\cdots=x^{(n-1)}=0$ уравнения (1.1), необходимо и достаточно, чтобы все корни уравнения

$$
\lambda^{n-2}+\sum_{i=1}^{n-1} a_{i} \lambda^{n-i-2}+a_{n-2}=0
$$

имели отрицательные вещественные части и, во-вторых, неравенства

$$
p>0, \quad q>0
$$

были удовлетворены.

Рассмотрим скалярное дифференциальное уравнение, соответствующее характеристическому уравнению (3.3)

$$
\begin{aligned}
x^{(n)}+\left(\alpha_{1}+p\right) x^{(n-1)}+\left(\alpha_{2}+p \alpha_{1}+q\right) x^{(n-2)}+ & \\
& +\sum_{j=3}^{n-2}\left(\alpha_{j}+p \alpha_{j-1}+q \alpha_{j-2}\right) x^{(n-j)}+\left(p \alpha_{n-2}+q \alpha_{n-3}\right) \dot{x}+q \alpha_{n-2} x=0,
\end{aligned}
$$

где коэффициенты $p, q$ и $\alpha_{i}, i=\overline{1, n-2}$, удовлетворяют условиям (3.4). 
Р. Предположим, что в уравнении $(3.7) p=p\left(x, \dot{x}, \ddot{x}, \ldots, x^{(n-1)}\right)-$ непрерывная положительная функция, обеспечивающая единственность решений, а коэффициенты $q$ и $\alpha_{i}$, $i=\overline{1, n-2},-$ постоянные числа. Предположим, что условие (3.6) удовлетворяется. Исследуем задачу устойчивости для решения $x=\dot{x}=\ddot{x}=\cdots=x^{(n-1)}=0$ уравнения (3.7).

Для решения этой проблемы перейдем от скалярного уравнения $n$-го порядка (3.7) к соответствующей системе $n$ уравнений первого порядка. Для этого сделаем замену переменных:

$$
\begin{gathered}
\dot{x}=x_{1}, \ddot{x}=x_{2}, \ldots, x^{(n-3)}=x_{n-3}, \\
x^{(n-2)}+\sum_{i=1}^{n-3} \alpha_{i} x^{n-i-2}+\alpha_{n-2} x=x_{n-2}, \\
x^{(n-1)}+\sum_{i=1}^{n-3} \alpha_{i} x^{n-i-1}+\alpha_{n-2} \dot{x}=x_{n-1} .
\end{gathered}
$$

Из первой группы уравнений (3.8) получаем следующие $n-3$ дифференциальных уравнений:

$$
\dot{x}=x_{1}, \dot{x}_{1}=x_{2}, \ldots, \dot{x}_{n-4}=x_{n-3} .
$$

Отсюда выводим дифференциальные уравнения относительно переменных $x_{n-2}$ и $x_{n-1}$.

Во-первых, заметим, что согласно (3.8) $\dot{x}_{n-2}=x_{n-1}$, откуда вытекает дифференциальное уравнение относительно $x_{n-2}$.

Во-вторых, нетрудно проверить, что представление $\dot{x}_{n-1}+p x_{n-1}+q x_{n-2}=0$ следует из уравнения (3.7). Это дает дифференциальное уравнение по переменной $x_{n-1}$.

Опираясь на предыдущие рассуждения и при условии, что $n>3$, получаем равенство

$$
x^{(n-2)}=-\sum_{i=1}^{n-3} \alpha_{i} x^{(n-i-2)}-\alpha_{n-2} x+x_{n-2}=-a_{n-2} x-\sum_{i=1}^{n-3} \alpha_{i} x_{n-i-2}+x_{n-2} .
$$

Заметим, что при $n=2$ и $n=3$ рассуждения также верны. Дифференцируя (3.9) по $t$, имеем

$$
x^{(n-1)}=\frac{d}{d t}\left(-\sum_{i=1}^{n-3} \alpha_{i} x_{n-i-2}-\alpha_{n-2} x+x_{n-2}\right)=-\sum_{i=1}^{n-3} \alpha_{i} x^{(n-i-1)}-\alpha_{n-2} \dot{x}+\dot{x}_{n-2} .
$$

В правую часть подставим выражения для производных (3.8) и (3.9). Тогда последовательно получим

$$
\begin{gathered}
x^{(n-1)}=-\alpha_{1} x^{n-2}-\sum_{i=2}^{n-3} \alpha_{i} x^{(n-i-1)}-\alpha_{n-2} \dot{x}+\dot{x}_{n-2}= \\
=-\alpha_{1}\left(-\alpha_{n-2} x-\sum_{i=1}^{n-3} \alpha_{i} x_{n-i-2}+x_{n-2}\right)-\sum_{i=2}^{n-3} \alpha_{i} x_{n-i-1}-\alpha_{i} x_{1}+x_{n-1}= \\
=\alpha_{1}\left(\alpha_{n-2} x+\alpha_{1} x_{n-3}\right)+\alpha_{1} \sum_{i=2}^{n-3} \alpha_{i} x_{n-i-2}-\alpha_{1} x_{n-2}-\sum_{i=1}^{n-3} \alpha_{i} x_{n-i-2}- \\
\quad-\alpha_{n-2} x_{1}+x_{n-1}=\alpha_{1} \alpha_{n-2} x-\alpha_{n-2} x_{1}+\alpha_{1} \sum_{i=2}^{n-3} \alpha_{i} x_{n-i-2}-
\end{gathered}
$$




$$
\begin{aligned}
-\sum_{i=1}^{n-3} \alpha_{i} x_{n-i-2} & +\left(\alpha_{1}\right)^{2} x_{n-3}-\alpha_{1} x_{n-2}+x_{n-1}=\alpha_{1} \alpha_{n-2} x-\alpha_{n-2} x_{1}+ \\
& +\alpha_{1} \sum_{i=2}^{n-3} \alpha_{i} x_{n-i-2}-\sum_{i=2}^{n-3} \alpha_{i} x_{n-i-2}-\alpha_{1} x_{n-3}+\left(\alpha_{1}\right)^{2} x_{n-3}-\alpha_{1} x_{n-2}+x_{n-1} .
\end{aligned}
$$

В качестве результата выводим формулу

$$
\begin{aligned}
x^{(n-1)}=\alpha_{1} \alpha_{n-2} x-\alpha_{n-2} x_{1}+\alpha_{1} \sum_{i=2}^{n-3} \alpha_{i} x_{n-i-2}- & \sum_{i=2}^{n-3} \alpha_{i} x_{n-i-2}- \\
& -\alpha_{1} x_{n-3}+\alpha_{1}\left(\alpha_{1}-1\right) x_{n-3}-\alpha_{1} x_{n-2}+x_{n-1} .
\end{aligned}
$$

Таким образом, исходное уравнение (3.7) с помощью замены переменных (3.8) переходит в систему $n$ дифференциальных уравнений первого порядка

$$
\begin{aligned}
& \dot{x}=x_{1}, \quad \dot{x}_{1}=x_{2}, \ldots, \dot{x}_{n-2}=x_{n-3}, \\
& \dot{x}_{n-3}=-\alpha_{n-1} x-\sum_{i=1}^{n-2} \alpha_{n-i-1} x_{i}+x_{n-1}, \\
& \dot{x}_{n-2}=x_{n-1}, \\
& \dot{x}_{n-2}=-q x_{n-2}-\varphi(X) x_{n-1} .
\end{aligned}
$$

Здесь для краткости $X=\left(x, x_{1}, x_{2}, \ldots, x_{n-1}\right)$ и функция

$$
\varphi(X)=p\left(x, \dot{x}, \ddot{x}, \ldots, x^{(n-1)}\right)=p\left(x, x_{1}, \ldots, x_{n-3},-\alpha n-2 x-\sum_{i=1}^{n-2} \alpha_{i} x_{i}+x_{n-2}, x^{(n-1)}\right)
$$

с аргументом $x^{(n-1)}$, определяемым установленной формулой (3.10).

Ясно, что проблема устойчивости нулевого решения $x=\dot{x}=\ddot{x}=\cdots=x^{(n-1)}=0$ дифференциального уравнения (3.7) эквивалентна проблеме устойчивости решения $x=$ $x_{1}=x_{2}=\cdots=x_{n-1}=0$ системы уравнений (3.11). Для исследования решений системы (3.11) возьмем знакопостоянную функцию

$$
V(X)=\frac{1}{2} q\left(x_{n-2}\right)^{2}+\frac{1}{2}\left(x_{n-1}\right)^{2} .
$$

Ее производная по времени вдоль решений (3.11) равна

$$
\dot{V}(X)=-\varphi(X)\left(x_{n-1}\right)^{2} .
$$

Устойчивостъ. Потребуем, чтобы выполнялись условия

$$
q>0, \quad \varphi(X) \geq 0 \text { для }\|X\|<h .
$$

Тогда функция $V$ является знакоположительной, а ее производная $\dot{V}-$ знакоотрицательной. На множестве $Y_{0}$, где $V(X)=0$, выполняются равенства $x_{n-2}=0, x_{n-1}=0$. Запишем систему (3.11) относительно множества $Y_{0}$. Это приводит к линейной системе

$$
\begin{aligned}
& \dot{x}=x_{1}, \quad \dot{x}_{1}=x_{2}, \ldots, \dot{x}_{n-2}=x_{n-3}, \\
& \dot{x}_{n-3}=-\alpha_{n-1} x-\sum_{i=1}^{n-2} \alpha_{n-i-1} x_{i} .
\end{aligned}
$$


Асимптотическая устойчивость нулевого решения (3.14) обеспечивается условиями РаусаГурвица [4] для полинома (3.5).

Следовательно, на основе теоремы 2.1 условия устойчивости нулевого решения системы (3.11) выполнены с учетом неравенства (3.13) и того факта, что все корни уравнения (3.5) имеют отрицательные вещественные части.

Асимптотическая устойчивостъ. Используем функцию (3.12). Согласно теореме 2.2 потребуем, чтобы на множестве, где производная равна нулю, нулевое решение обладало бы свойством асимптотической устойчивости. Для этого потребуем выполнение следующих неравенств:

$$
q>0, \quad \varphi(X)>0 \text { при }\|X\|<h .
$$

Тогда на множестве $Y$ имеем равенства $x_{n-2}=0, x_{n-1}=0$. Здесь система переходит в (3.14). Следовательно, теорема 2.2 гарантирует асимптотическую устойчивость нулевого решения системы (3.9) при выполнении (3.13).

Глобальная асимптотическая устойчивость. Предположим, что условия (3.15) выполнены для любого $h>0$ и все корни уравнения (3.5) имеют отрицательные действительные части. Используем знакопостоянную функцию (3.12) и покажем, что каждое решение $\left(x(t), x_{1}(t), x_{2}(t), \ldots, x_{n-1}(t)\right)$ системы $(3.11)$ ограничено для всех $t>0$.

Так как $q>0$, то наличие знакоположительной функции (3.12) со знакоотрицательной производной по времени влечет ограниченность решения по координатам $\left(x_{n-2}(t), x_{n-1}(t)\right)$. Покажем ограниченность и по остальным координатам.

Рассмотрим систему

$$
\begin{aligned}
& \dot{x}=x_{1}, \quad \dot{x}_{1}=x_{2}, \ldots, \dot{x}_{n-2}=x_{n-3}, \\
& \dot{x}_{n-3}=-\alpha_{n-1} x-\sum_{i=1}^{n-2} \alpha_{n-i-1} x_{i}+x_{n-1}(t),
\end{aligned}
$$

где $x_{n-1}(t)$ - компонента $\left(x(t), x_{1}(t), x_{2}(t), \ldots, x_{n-1}(t)\right)$. Согласно предположению об асимптотической устойчивости нулевого решения системы (3.14) следует, что оно и глобально асимптотически устойчиво с учетом линейности системы. Кроме того, для системы (3.16) выполнены все условия леммы 2.1, откуда и следует ограниченность по отношению к $\left(x(t), x_{1}(t), x_{2}(t), \ldots, x_{n-1}(t)\right)$.

Таким образом, на основании теоремы 2.3 условия

$$
q>0, \varphi(X)>0 \text { для всех } X \in \mathbb{R}^{n}
$$

дают глобальную асимптотическую устойчивость нулевого решения системы (3.11). Исследования показывают, что можно сформулировать и свойство неустойчивости, пользуясь замечанием 2.1.

Теорема 3.1. Предположим, что все корни уравнения (3.5) имеют отрицательные действительные части и $q>0$. Тогда нулевое решение уравнения (3.7) будет

1) устойчивым, если для некоторого $h>0$

$$
p\left(x, \dot{x}, \ddot{x}, \ldots, x^{(n-1)}\right) \geq 0 \quad \text { nрu }\left\|\left(x, \dot{x}, \ddot{x}, \ldots, x^{(n-1)}\right)\right\|<h ;
$$

2) асимптотически устойчивым, если для некоторого $h>0$

$$
p\left(x, \dot{x}, \ddot{x}, \ldots, x^{(n-1)}\right)>0 \quad n p u \quad\left\|\left(x, \dot{x}, \ddot{x}, \ldots, x^{(n-1)}\right)\right\|<h ;
$$

3) глобально асимптотически устойчивым, если

$$
p\left(x, \dot{x}, \ddot{x}, \ldots, x^{(n-1)}\right)>0 \quad \text { для всех }\left(x, \dot{x}, \ddot{x}, \ldots, x^{(n-1)}\right) \in \mathbb{R}^{n} .
$$


Если для уравнения (3.5) существует коренъ с положительной вещественной частью, то нулевое решение уравнения (3.7) неустойчиво.

2. Случай действительных корней. Предположим, что характеристическое уравнение (3.1) имеет действительный корень $\lambda=-s$. (Если $n-$ нечетное число, то это всегда возможно). В этом случае (3.1) можно записать в форме

$$
\left(\lambda^{n-1}+\sum_{i=1}^{n-2} \alpha_{i} \lambda^{n-i-1}+\alpha_{n-1}\right)(\lambda+s)=0 .
$$

Перемножая скобки в (3.17), получим уравнение

$$
\lambda^{n}+\left(\alpha_{1}+s\right) \lambda^{n-1}+\sum_{i=2}^{n-1}\left(\alpha_{i}+s \alpha_{i-1}\right) \lambda^{n-i}+s \alpha_{n-1}=0
$$

Более того, сравнивая представления (1.1) и (3.17), можем записать

$$
a_{1}=\alpha_{1}+s, \quad a_{n}=s \alpha_{n-1}, \quad a_{i}=\alpha_{i}+s \alpha_{i-1}, \quad i=\overline{2, n-1} .
$$

Таким образом, при наличии действительного корня $\lambda=-s$ дифференциальное уравнение (1.1) может быть записано в виде

$$
x^{(n)}+\left(\alpha_{1}+s\right) x^{(n-1)}+\sum_{i=2}^{n-1}\left(\alpha_{i}+s \alpha_{i-1}\right) x^{(n-i)}+s \alpha_{n-1} x=0,
$$

где коэффициенты $s$ и $\alpha_{i}, i=\overline{1, n-1}$, удовлетворяют уравнениям (3.19).

S. Рассмотрим проблему устойчивости дифференциального уравнения (3.20) при условии, что $s=s\left(x, \dot{x}, \ddot{x}, \ldots, x^{(n-1)}\right)-$ непрерывная функция, обеспечивающая единственность решений.

Для решения проблемы используем новые переменные в (3.20):

$$
\dot{x}=x_{1}, \ddot{x}=x_{2}, \ldots, x^{(n-2)}=x_{n-2} ; x^{(n-1)}+\sum_{i=1}^{n-2} \alpha_{i} x^{(n-i-1)}+\alpha_{n-1} x=x_{n-1} .
$$

Из первой группы уравнений получаем $n-2$ дифференциальных уравнений первого порядка

$$
\dot{x}=x_{1}, \dot{x}_{1}=x_{2}, \ldots, \dot{x}_{n-3}=x_{n-2} .
$$

Кроме того, очевидно, что в силу дифференциального уравнения (3.20) верно равенство $\dot{x}_{n-1}=-s x_{n-1}$. Это, в частности, дает дифференциальное уравнение относительно переменной $x_{n-1}$. Далее, дифференцируя последнее равенство в (3.21), получаем

$$
\frac{d x^{(n-1)}}{d t}+\sum_{i=1}^{n-2} \alpha_{i} x^{(n-i)}+\alpha_{n-1} \dot{x}=\dot{x}_{n-1} .
$$

Следовательно, имеем

$$
\begin{array}{r}
\dot{x}_{n-2}=-s x_{n-1}-\sum_{i=1}^{n-2} \alpha_{i} x^{(n-i)}-\alpha_{n-1} x_{1}= \\
-s x_{n-1}-\alpha_{1} x_{1}-\sum_{i=2}^{n-2} \alpha_{i} x_{n-i}-\alpha_{n-1} x_{1}= \\
=-\alpha_{n-1} x_{1}-\sum_{i=2}^{n-2} \alpha_{i} x_{n-i}-\left(s+\alpha_{1}\right) x_{n-1} .
\end{array}
$$


Таким образом, исходное дифференциальное уравнение $n$-го порядка (3.20) с помощью замены переменных (3.21), (3.22) переходит к системе $n$ уравнений первого порядка

$$
\begin{aligned}
& \dot{x}=x_{1}, \quad \dot{x}_{1}=x_{2}, \ldots, \dot{x}_{n-3}=x_{n-2}, \\
& \dot{x}_{n-2}=-\alpha_{n-1} x_{1}-\sum_{i=2}^{n-2} \alpha_{i} x_{n-i}-\left(s+\alpha_{1}\right) x_{n-1}, \\
& \dot{x}_{n-1}=-\varphi(X) x_{n-1},
\end{aligned}
$$

где $X=\left(x, x_{1}, x_{2}, \ldots, x_{n-1}\right)$ и

$$
\varphi(X)=s\left(x, \dot{x}, \ddot{x}, \ldots, x^{(n-1)}\right)=s\left(x, x_{1}, x_{2}, \ldots, x_{n-2},-\alpha_{n-1} x_{1}-\sum_{i=2}^{n-2} \alpha_{i} x_{n-i}-\left(s+\alpha_{1}\right) x_{n-1}\right) .
$$

Для исследования проблемы устойчивости этой системы возьмем знакоположительную функцию со знакоотрицательной производной по времени в силу системы (3.23):

$$
V(X)=\frac{1}{2}\left(x_{n-1}\right)^{2}, \quad \dot{V}(X)=-\varphi(X)\left(x_{n-1}\right)^{2} .
$$

Устойчивостъ. Потребуем выполнение условия

$$
\varphi(X) \geq 0 \text { при }\|X\|<h .
$$

Тогда производная $\dot{V}$ будет иметь отрицательный знак. На множестве $Y_{0}$, где $V(X)=0$ выполняется равенство $x_{n-1}=0$. Запишем систему (3.23) относительно множества $Y_{0}$. Она редуцируется в простую линейную систему

$$
\begin{aligned}
& \dot{x}=x_{1}, \quad \dot{x}_{1}=x_{2}, \ldots, \dot{x}_{n-3}=x_{n-2}, \\
& \dot{x}_{n-2}=-\alpha_{n-1} x_{1}-\sum_{i=2}^{n-2} \alpha_{i} x_{n-i}-\left(s+\alpha_{1}\right) x_{n-1} .
\end{aligned}
$$

Асимптотическая устойчивость нулевого решения (3.26) определяется критерием РаусаГурвица для полинома

$$
\lambda^{n-1}+\sum_{i=1}^{n-2} \alpha_{i} \lambda^{n-i-1}+\alpha_{n-1}=0 .
$$

Следовательно, на основании теоремы 2.1 условия устойчивости нулевого решения системы (3.23) определяются неравенством (3.25) и тем фактом, что все корни уравнения (3.27) имеют отрицательные действительные части.

Асимптотическая устойчивостъ. Можно показать, что при выполнении неравенства

$$
\varphi(X)>0 \text { при }\|X\|<h
$$

и требования того, что все корни уравнения (3.27) имеют отрицательные действительные части, получаем асимптотическую устойчивость нулевого решения системы (3.23).

Глобальная асимптотическал устойчивость. Предположим, что условия (3.25) выполнены для любого $h>0$ и все корни уравнения (3.27) имеют отрицательные действительные части. Используем знакопостоянную функцию (3.24) и покажем, что каждое решение $\left(x(t), x_{1}(t), x_{2}(t), \ldots, x_{n-1}(t)\right)$ системы (3.23) ограничено при $t>0$. Действительно, так как $s>0$, наличие знакоположительной функции (3.24) со знакоотрицательной производной по времени показывает, что решение $\left(x(t), x_{1}(t), x_{2}(t), \ldots, x_{n-1}(t)\right)$ системы $(3.23)$ ограничено 
по координате $x_{n-1}(t)$. Покажем, что по остальным координатам решение также ограничено.

Рассмотрим систему

$$
\begin{aligned}
& \dot{x}=x_{1}, \quad \dot{x}_{1}=x_{2}, \ldots, \dot{x}_{n-3}=x_{n-2}, \\
& \dot{x}_{n-2}=-\alpha_{n-1} x_{1}-\sum_{i=2}^{n-2} \alpha_{i} x_{n-i}-\left(s+\alpha_{1}\right) x_{n-1}(t),
\end{aligned}
$$

где $x_{n-1}(t)$ - компонента $\left(x(t), x_{1}(t), x_{2}(t), \ldots, x_{n-1}(t)\right)$. Нетрудно видеть, что для системы (3.28) все условия леммы 2.1 выполнены, откуда получаем ограниченность по координатам $\left(x(t), x_{1}(t), x_{2}(t), \ldots, x_{n-1}(t)\right)$.

Таким образом, на основании теоремы 2.3 условия $\varphi(X)>0$ для всех $X \in \mathbb{R}^{n}$ дают глобальную асимптотическую устойчивость нулевого решения системы (3.23).

Теорема 3.2. Предположим, ито все корни уравнения (3.27) имеют отрицательные действительные части. Тогда нулевое решение уравнения (3.20) является

1) устойчивым, если для некоторого $h>0$

$$
s\left(x, \dot{x}, \ddot{x}, \ldots, x^{(n-1)}\right) \geq 0 \quad n p u \quad\left\|\left(x, \dot{x}, \ddot{x}, \ldots, x^{(n-1)}\right)\right\|<h ;
$$

2) асимптотически устойчивым, если для некоторого $h>0$

$$
s\left(x, \dot{x}, \ddot{x}, \ldots, x^{(n-1)}\right)>0 \quad n p u \quad\left\|\left(x, \dot{x}, \ddot{x}, \ldots, x^{(n-1)}\right)\right\|<h ;
$$

3) глобально асимптотически устойчивым, если

$$
s\left(x, \dot{x}, \ddot{x}, \ldots, x^{(n-1)}\right)>0 \quad \text { для всех }\left(x, \dot{x}, \ddot{x}, \ldots, x^{(n-1)}\right) \in \mathbb{R}^{n} .
$$

Если в уравнении (3.27) имеется корень с положительной действительной частью, то нулевое решение уравнения (3.20) неустойчиво.

Пример 3.1. Рассмотрим скалярное дифференциальное уравнение второго порядка

$$
\ddot{x}+(f(x, \dot{x})+b) \dot{x}+b f(x, \dot{x}) x=0,
$$

где $f: \mathbb{R}^{2} \rightarrow \mathbb{R}$ - непрерывная функция, удовлетворяющая некоторым условиям, обеспечивающим единственность решений. Соответствующее линейное дифференциальное уравнение есть $\ddot{x}+(a+b) \dot{x}+a b=0$. Его характеристическое уравнение $\lambda^{2}+(a+b) \lambda+a b=0$ имеет действительные корни

$$
\lambda_{1}=-a, \quad \lambda_{2}=-b .
$$

Для асимптотической устойчивости линейного дифференциального уравнения необходимо и достаточно, чтобы выполнялись неравенства $a>0, b>0$. Согласно теореме 2.3 решение $x=\dot{x}=0$ уравнения (3.29) будет глобально асимптотически устойчивым, если выполняются условия

$$
b>0, \quad f(x, \dot{x})>0 \text { для всех }(x, \dot{x}) \in \mathbb{R}^{2} .
$$

Уравнение (3.29) имеет тип уравнения Льенара [15]. Исследование проблемы Айзермана для уравнения типа уравнение Льенара изучалось также в работе автора [16]. 


\section{ЗАКЛЮЧЕНИЕ}

1) Утверждения теорем 3.1 и 3.2 о глобальной асимптотической устойчивости показывают, что задача Айзермана для скалярного дифференциального уравнения (1.1) имеет положительное решение по параметру, обозначающему действительную часть корня его характеристического уравнения (параметр $p$ для системы (3.9) и параметр $s$ для системы (3.23)).

2) Достаточные условия глобальной асимптотической устойчивости, полученные в теоремах 3.1 и 3.2 , совпадают с необходимыми и достаточными условиями асимптотической устойчивости в соответствующем линейном случае скалярного дифференциального уравнения (1.1). Это подчеркивает принадлежность к общепринятой качественной оценке результатов, полученных для задачи об асимптотической устойчивости равновесия для любых начальных возмущений.

3) В каждом рассматриваемом случае построение знакопостоянной функции $V$ для решения проблем устойчивости является достаточно простым делом, и совершенно неясно, что поиск соответствующей классической знакоопределенной функции Ляпунова приведет к положительному результату.

4) Нетрудно проверить, что требования теорем 3.1 и 3.2 относительно глобальной асимптотической устойчивости не совпадают с соответствующими условиями [9] для скалярного дифференциального уравнения вида (3.29).

\section{ЛитератУРА}

[1] Айзерман М.А. Об одной проблеме, касающейся устойчивости "в большом" динамических систем, Усп. матем. наук 4 (4), 187-188 (1949).

[2] Еругин Н.П. О некоторых вопросах устойчивости движения и качественной теории дифберенциалъных уравнений в иелом, Прикл. матем. и механ. 14 (5), 459-512 (1950).

[3] Еругин Н.П. Некоторье общие вопросъ теории устойчивости движения, Прикл. матем. и механ. 15 (2), 227-236 (1951).

[4] Малкин И.Г. Теория устойчивости движения (Наука, М., 1966).

[5] Красовский Н.Н. Теоремы об устойчивости движения, определяемых системой двух уравнений, Прикл. матем. и механ. 16 (3), 546-554 (1952).

[6] Плисс В.А. Некоторые проблемъ теории устойчивости движения в целом (Изд. ЛГУ, Ленинград, 1958).

[7] Леонов Г.А. О проблеме Айзермана, Автомат. и телемехан., № 7, 37-49 (2009).

[8] Hahn W. Stability of motion (Springer-Verlag, New York, 1967).

[9] Барбашин Е.А. Функции Ллпунова (Наука, М., 1970).

[10] Калитин Б.С. Устойчивость дифференциалънъх уравнений (Метод знакопостолнных функций Ллпунова) (LAP Lambert Academic Publishing, Saarbrücken, 2012).

[11] Руш Н., Абетс П., Лалуа М. Прямой метод Ляпунова в теории устойчивости (Мир, М., 1980).

[12] Булгаков Н.Г., Калитин Б.С. Обобщение теорем второго метода Ллпунова. І. Теория, Изв. АН БССР. Сер. физ.-матем. наук, № 3, 32-36 (1978).

[13] Калитин Б.С. Устойчивостъ неавтономных дифференциалъных уравнений (БГУ, Минск, 2013).

[14] Демидович Б.П. Лекиии по математической теории устойчивости (Наука, М., 1967).

[15] Liénard A. Étude des oscillations autoentretienes, Rev. Gen. Elec. 23, 901-902, 946-954 (1928).

[16] Калитин Б.С. Об устойчивости уравнения Лъенара, Изв. вузов. Матем., № 10, 17-28 (2018).

\section{Борис Сергеевич Калитин}

Белорусский государственный университет, пр-т Независимости, д. 4, г. Минск, 220030, Республика Беларусь,

e-mail: Kalitine@yandex.ru 


\section{B.S. Kalitine}

\section{On the Aizerman problem for the scalar differential equations}

Abstract. We deal with the problem of stability of the equilibrium of a $n$-th order scalar differential equation. A positive solution is obtained for the Aizerman problem for equations of a special type. We have proved that the parameter of the real part of root of the characteristic equation can be replaced by an arbitrary continuous function depending on all phase variables while preserving the properties of global asymptotic stability.

Keywords: scalar differential equation, equilibrium, stability, Lyapunov functions.

Boris Sergeevich Kalitine

Belorussian State University,

4 Nezavisimosti Ave., Minsk, 220030 Republic of Belarus,

e-mail: Kalitine@yandex.ru 